\title{
Point-of-care ultrasound in the management of shock: what is the optimal prescription?
}

\author{
Kyle Fiorini $\cdot$ John Basmaji
}

Received: 6 October 2021/Revised: 6 October 2021/ Accepted: 6 October 2021/Published online: 24 November 2021

(C) Canadian Anesthesiologists' Society 2021

The management of shock can be a daunting endeavour for even the most seasoned front-line resuscitationists. Patients in shock present with similar signs and symptoms despite having different physiologic derangements underlying their clinical presentation. In addition, various shock subtypes can have competing therapeutic goals, leaving clinicians with a small margin for error when making decisions. It is of no surprise then that resuscitationists have gravitated towards hemodynamic guidance systems that can unravel cryptic shock physiology directly at the bedside (i.e., pointof-care). Such hemodynamic guidance systems have become deeply entrenched in the culture and practice of resuscitation medicine. ${ }^{1}$

Over the years, several point-of-care hemodynamic monitoring systems have come in and out of favour, but not one device has earned tenure. ${ }^{2}$ Point-of-care ultrasound (POCUS), one of the latest iterations of such hemodynamic guidance systems, has gained widespread adoption among acute care physicians in the past decade. ${ }^{3}$ Point-of-care ultrasound has several traits that set it apart from other hemodynamic guidance systems. First, it provides rapid and dynamic visualization of physiology for various organs, including the heart, lungs, vascular system, and abdomen. Furthermore, it is broadly accessible across various practice settings, scalable to a broad user base, and is non-invasive to patients. ${ }^{4}$ These merits have granted POCUS tenancy in intensive care units, emergency rooms, and operating rooms, for the time being. 5,6

K. Fiorini $(\bowtie) \cdot$ J. Basmaji $(\bowtie)$

Division of Critical Care, Department of Medicine, London

Health Sciences Centre, London, ON, Canada

e-mail: kfiorini2017@meds.uwo.ca

e-mail: jbasmaji@uwo.ca
The clinical use of POCUS, however, has burgeoned at a rate that has outpaced its own evidence base. ${ }^{7}$ This phenomenon is likely due to the challenges associated with studying point-of-care hemodynamic guidance systems that straddle the domains of diagnostic tools and therapeutic interventions. Like its predecessors, POCUS remains an inherently diagnostic tool that classifies patients by disease states (e.g., hypovolemic or cardiogenic shock). Nevertheless, as a hemodynamic guidance system, POCUS also identifies patients who are appropriate recipients of specific treatments (e.g., patients who are deemed 'volume responsive' or those who are candidates for positive inotropes) and therefore becomes complicit as part of a therapeutic strategy. We believe that POCUS ought to be studied primarily as an intervention, with research programs first vetting its diagnostic faculties before proceeding to study its therapeutic impact.

The current body of evidence has already established an important "proof-of-concept" milestone: POCUS exhibits strong diagnostic accuracy for determining the cause of shock and circulatory failure. ${ }^{8}$ How, then, do we apply this tool in a way that efficiently harnesses the relevant diagnostic datapoints, empowers the clinician to prescribe the most effective therapies, and improves patient outcomes? Parsing these questions further, we must determine: (1) how early in the patient's clinical course-and how often-do we apply POCUS; (2) the minimum competency standards we require of POCUS users; and (3) the optimal scope of POCUS with respect to the number of views, the complexity of the scans, and the number of organ systems we should evaluate. Addressing these knowledge gaps will allow us to conceptualize a "dose-response" relationship for the provision of POCUS, while balancing concepts of "efficacy" (comprehensive POCUS assessments done by expert users) and 
"effectiveness" (pragmatic, goal-directed POCUS assessments performed by a broad user base).

In this issue of the Journal, Bughrara et al. report the results of a study that is germane to the aforementioned knowledge gaps in this field of research. ${ }^{9}$ The authors enrolled adult patients with hemodynamic instability, respiratory failure, or undefined volume status in the noncardiac perioperative setting and compared two different applications of POCUS. They evaluated how well a single subcostal view plus inferior vena cava assessment (echocardiographic assessment using the subcostal-only [or EASy] view) compared with focused transthoracic echocardiography (FTTE) in the hands of novice users. In their study, users were given $20 \mathrm{~h}$ of instruction, delivered through a combination of didactic teaching, hands-on training, and high-fidelity simulation. Then, images from 102 patients were acquired by novice users and independently reviewed by two experts. From their results, EASy assessment showed high agreement with FTTE when assessing for the presence of pericardial effusion (Gwet's AC1, 0.98; 95\% confidence interval [CI], 0.95 to 1.0 ), right ventricular contractility (Gwet's AC1, $0.84 ; 95 \% \mathrm{CI}, 0.74$ to 0.95$)$, and interventricular septal motion (Gwet's AC1, 0.92; 95\% CI, 0.85 to 0.99). There was moderate to substantial agreement for assessing right ventricular size (Gwet's AC1, 0.70; 95\% CI, 0.54 to 0.85), left ventricular size (Gwet's AC1, 0.73; 95\% CI, 0.58 to 0.88 ), and left ventricular contractility (Gwet's AC1, 0.73, $95 \% \mathrm{CI} ; 0.58$ to 0.88$)$. The authors concluded that a subcostal only approach, when it could be obtained, provides valuable diagnostic information sufficient to aid in the management of patients with cardiopulmonary derangements.

We commend the authors on their work to better refine the minimum competency standards of the operator and the scope of POCUS that still yields valuable clinical information, all while reducing the time required to complete the scan. The EASy assessment, despite being a reductionistic take on FTTE, still enabled novice clinicians to identify "actionable" pathologies that are amenable to life-saving interventions, such as draining a pericardial effusion, prescribing thrombolytic therapy, or administering inotropes. Distilling POCUS protocols down to their most useful constituents will reduce the barrier to entry for most users, while preserving their diagnostic faculties and capacity to guide appropriate interventions. Furthermore, the EASy assessment was obtained by novice ultrasonographers in $4 \mathrm{~min}-\mathrm{a}$ marked reduction in time compared with the FTTE examination, which required $16 \mathrm{~min}$. Curtailing the time required to undertake POCUS scans mitigates a potential shortcoming of this technology; serial hemodynamic evaluations using POCUS require dedicated time and undivided attention at the expense of the clinician. If POCUS protocols become too onerous, enthusiasm for this modality may wane, particularly in short-staffed, high patient census settings such as emergency departments or intensive care units. This is in contrast to other hemodynamic guidance systems like the pulmonary artery catheter, where, once the device is inserted, hemodynamic data are continuously obtained without taxing the clinician's time any further.

The authors' work raises several important questions. First, image interpretation was performed by expert users, who may require fewer data points than novice users to identify "actionable" pathology and confidently implement therapies under duress. In addition, up to $20 \%$ of scans were of poor quality and not interpretable, potentially overestimating the benefit of the EASy assessment. Finally, Gwet's agreement coefficient reported by the authors only informs us of incongruence between the EASy assessment and FTTE in aggregate, without providing granular data on whether disagreements occurred when "ruling in" or "ruling out" of pathology.

The findings of this study contribute to an important academic conversation on what the optimal prescription of POCUS ought to be, with respect to the user competency and the scope of POCUS (e.g., the number of views and the complexity of the scans). Bughrara et al. demonstrated that a subcostal only approach is an effective introductory point for learners, but we cannot solely rely on this view for a significant number of patients. While the subcostal view may be the most appropriate first step in time-sensitive resuscitations, we expect most clinicians will follow through with a more comprehensive echocardiographic examination, affording users more incremental information through the acquisition of serial cardiac views. Future work in this area should further refine the user competency standards and scope of POCUS such that its application is efficient, rapid, and accessible, without compromising its diagnostic prowess and therapeutic impact.

\section{L'échographie ciblée pour la prise en charge du choc: quelle est la prescription optimale?}

La prise en charge des chocs peut être intimidante et ce, même pour les réanimateurs de première ligne les plus chevronnés. Les patients en état de choc présentent des signes et des symptômes similaires malgré des dérangements physiologiques différents sous-jacents à leur présentation clinique. En outre, divers sous-types de 
choc peuvent avoir des objectifs thérapeutiques opposés, laissant aux cliniciens une très faible marge d'erreur lors de la prise de décision. Il n'est donc pas surprenant que les réanimateurs se soient tournés vers des systèmes de surveillance hémodynamique qui peuvent déchiffrer la physiologie d'un choc cryptique directement au chevet du patient (c.-à-d. au point de service ou ciblé). Ces systèmes de monitorage hémodynamique sont devenus profondément ancrés dans la culture et la pratique de la médecine de réanimation. ${ }^{1}$

$\mathrm{Au}$ fil des ans, plusieurs systèmes de monitorage hémodynamique de proximité ont eu leur moment de gloire et leur chute dans l'oubli, mais aucun n'est véritablement resté pour devenir l'étalon or. ${ }^{2} \mathrm{Au}$ cours de la dernière décennie, l'échographie ciblée (ou POCUS, pour Point-Of-Care UltraSound), l'une des plus récentes itérations de ces systèmes de monitorage hémodynamique, a été largement adoptée par les médecins de soins aigus. ${ }^{3}$ L'échographie ciblée présente plusieurs caractéristiques qui la distinguent des autres systèmes de monitorage hémodynamique. Tout d'abord, elle permet une visualisation rapide et dynamique de la physiologie de divers organes, dont le cœur, les poumons, le système vasculaire et l'abdomen. En outre, elle est largement accessible dans divers milieux de pratique, est adaptable à une importante base d'utilisateurs et est non invasive pour les patients. ${ }^{4}$ Grâce à ces caractéristiques avantageuses, l'échographie ciblée s'est mérité une place de choix dans l'arsenal des unités de soins intensifs, des salles d'urgence et des salles d'opération, pour le moment du moins. ${ }^{5,6}$

L'utilisation clinique de l'échographie ciblée, cependant, a connu un essor à un rythme qui a dépassé sa propre base de données probantes. ${ }^{7}$ Ce phénomène est probablement dû aux défis associés à l'étude des systèmes de monitorage hémodynamique de proximité qui appartiennent à la fois au domaine des outils diagnostiques et à celui des interventions thérapeutiques. Tout comme ses prédécesseurs, l'échographie ciblée demeure fondamentalement un outil de diagnostic qui permet de classer les patients selon leur état pathologique (par exemple, choc hypovolémique ou cardiogénique). Néanmoins, en tant que système de monitorage hémodynamique, l'échographie ciblée permet également l'identification des patients qui seraient de bons candidats à certains traitements spécifiques (par exemple, les patients qui sont considérés comme «répondant bien au traitement volémique» ou ceux qui sont candidats à recevoir des inotropes positifs) et devient donc une composante qui a sa place dans le cadre d'une stratégie thérapeutique. Selon nous, l'échographie ciblée devrait être étudiée principalement en tant qu'intervention, et des programmes de recherche devraient d'abord vérifier et valider ses capacités diagnostiques avant de procéder à l'étude de son impact thérapeutique.

L'ensemble des données probantes actuelles a déjà établi une étape importante de la «démonstration de faisabilité»: l'échographie ciblée présente une grande précision diagnostique pour déterminer la cause du choc et de l'insuffisance circulatoire. ${ }^{8}$ Comment, alors, appliquer cet outil de manière à exploiter efficacement les points de données diagnostiques pertinents, à permettre au clinicien de prescrire les traitements les plus efficaces et à améliorer les issues des patients? En analysant davantage ces questions, nous devons déterminer: (1) à quel stade (plus ou moins tôt) de l'évolution clinique du patient - et à quelle fréquence - nous devrions utiliser l'échographie ciblée; (2) les normes minimales de compétence à exiger des utilisateurs de l'échographie ciblée; et (3) la portée optimale de l'échographie ciblée en ce qui concerne le nombre de vues, la complexité des images et le nombre de systèmes d'organes que nous devrions évaluer. En comblant ces lacunes dans les connaissances, nous pourrons conceptualiser une relation de «dose-réponse» pour la fourniture de l'échographie ciblée, tout en équilibrant les concepts d' «efficacité» (évaluations d'échographie ciblées complètes réalisées par des utilisateurs experts) et d'«efficience» (évaluations pragmatiques et axées sur des objectifs de l'échographie ciblée réalisées par une large base d'utilisateurs).

Dans ce numéro du Journal, Bughrara et coll. rendent compte des résultats d'une étude pertinente aux lacunes susmentionnées dans les connaissances dans ce domaine de recherche. ${ }^{9}$ Les auteurs ont recruté des patients adultes présentant une instabilité hémodynamique, une insuffisance respiratoire ou une volémie indéfinie dans un contexte périopératoire non cardiaque et ont comparé deux applications différentes de l'échographie ciblée. Ils ont évalué dans quelle mesure une vue sous-costale unique, complétée par une évaluation de la veine cave inférieure (évaluation échocardiographique utilisant la vue souscostale unique [ou EASy]), se comparait à l'échocardiographie transthoracique ciblée (ETTC) entre les mains d'utilisateurs novices. Dans leur étude, les utilisateurs ont reçu 20 heures de formation, dispensées grâce à une combinaison d'enseignement didactique, de formation pratique et de simulation haute fidélité. Ensuite, les images de 102 patients ont été acquises par des utilisateurs novices et indépendamment examinées par deux experts. D'après leurs résultats, l'évaluation EASy a montré une concordance élevée avec l'ETTC lors de l'examen pour évaluer la présence d'un épanchement péricardique (AC1 de Gwet, 0.98; intervalle de confiance [IC] à $95 \%, 0.95$ à 1.0), la contractilité ventriculaire droite (AC1 de Gwet, 0.84; IC 95\%, 0.74 à 0.95) et le mouvement septal interventriculaire (AC1 de Gwet, 0.92; IC 95\%, 0.85 
à 0.99). La concordance était modérée à substantielle en ce qui touchait à l'évaluation de la taille du ventricule droit (AC1 de Gwet, 0.70; IC 95\%, 0.54 à 0.85), de la taille du ventricule gauche (AC1 de Gwet, 0.73; IC 95\%, 0.58 à 0.88 ) et de la contractilité ventriculaire gauche (ACA de Gwet, 0.73, IC 95\%; 0.58 à 0.88). Les auteurs ont conclu qu'une approche unique sous-costale, lorsqu'elle pouvait être obtenue, fournissait des informations diagnostiques précieuses suffisantes pour aider à la prise en charge des patients présentant des troubles cardiopulmonaires.

Nous félicitons les auteurs pour leurs travaux visant à mieux préciser les normes minimales de compétence de l'opérateur et la portée de l'échographie ciblée qui fournit toujours des informations cliniques précieuses, tout en réduisant le temps nécessaire pour terminer l'examen. L'évaluation EASy, bien qu'en soi une version réductionniste de l'ETTC, a tout de même permis aux cliniciens novices d'identifier des pathologies «concrètes» se prêtant à des interventions vitales, telles que le drainage d'un épanchement péricardique, la prescription d'un traitement thrombolytique ou encore l'administration d'inotropes. La cristallisation des protocoles d'échographie ciblée jusqu'à leurs éléments les plus utiles réduira l'obstacle d'accessibilité pour la plupart des utilisateurs, tout en préservant les facultés de diagnostic et la capacité à guider des interventions adaptées de cette modalité. De plus, l'évaluation EASy a pu être réalisée par des échographistes novices en 4 minutes - une réduction marquée du temps par rapport à l'examen d'ETTC, qui a nécessité $16 \mathrm{~min}$. La réduction du temps nécessaire pour réaliser des examens d'échographie ciblée atténue un défaut potentiel de cette technologie: en effet, les évaluations hémodynamiques en série utilisant l'échographie ciblée nécessitent du temps dédié et une attention entière et complète du clinicien. Si les protocoles d'échographie ciblée deviennent trop lourds, l'enthousiasme pour cette modalité pourrait s'étioler, en particulier dans les contextes à court de personnel et où le nombre de patients est élevé, tels que les départements d'urgence ou les unités de soins intensifs. Cela contraste avec d'autres systèmes de monitorage hémodynamique tel que le cathéter de l'artère pulmonaire, où, une fois le dispositif positionné, les données hémodynamiques sont obtenues en continu - et ce, sans nécessiter plus de temps du clinicien.

Le travail des auteurs soulève plusieurs questions importantes. Tout d'abord, l'interprétation des images a été réalisée par des utilisateurs experts, lesquels ont probablement besoin de moins de points de données que des utilisateurs novices pour identifier la pathologie «traitable» et initier avec assurance des traitements, même en situation d'urgence. De plus, jusqu'à $20 \%$ des examens étaient de mauvaise qualité et non interprétables, ce qui pourrait surestimer les avantages d'une évaluation EASy. Enfin, le coefficient de concordance Gwet rapporté par les auteurs ne fait que nous informer de l'incongruité entre l'évaluation EASy et l'ETTC dans son ensemble, sans fournir de données détaillées sur la question de savoir si des désaccords se sont produits lors de «l'inclusion possible» ou de «l'exclusion» de la pathologie.

Les résultats de cette étude contribuent à une importante discussion académique sur ce que devrait être la prescription optimale d'échographie ciblée, au regard de la compétence de l'utilisateur et de la portée de l'échographie ciblée (par exemple, le nombre de vues et la complexité des examens). Bughrara et coll. ont démontré qu'une approche sous-costale unique constituait un point d'entrée efficace pour les apprenants, mais nous ne pouvons pas nous fonder uniquement sur cette vue pour un nombre considérable de patients. Bien que la vue souscostale puisse constituer la première étape la plus adaptée lors d'une réanimation urgente, la plupart des cliniciens approfondiront probablement leur analyse avec un examen échocardiographique plus poussé, offrant aux utilisateurs davantage d'informations incrémentielles grâce à l'acquisition de vues cardiaques en série. Les travaux futurs dans ce domaine devraient s'efforcer de préciser davantage les normes de compétence des utilisateurs et la portée de l'échographie ciblée afin que son application soit efficace, rapide et accessible, sans compromettre ses prouesses diagnostiques et son impact thérapeutique.

\section{Conflict of interest None.}

Funding None.

Editorial responsibility This submission was handled by Dr. Philip M. Jones, Deputy Editor-in-Chief, Canadian Journal of Anesthesia.

Déclaration Aucune.

Déclaration de financement Aucune.

Responsabilité éditoriale Cet article a été traité par Dr Philip M. Jones, rédacteur en chef adjoint, Journal canadien d'anesthésie.

\section{References}

1. Vincent JL, Rhodes A, Perel A, et al. Clinical review: update on hemodynamic monitoring - a consensus of 16. Crit Care 2011. DOI: https://doi.org/10.1186/cc10291.

2. Huygh J, Peeters Y, Bernards J, Malbrain ML. Hemodynamic monitoring in the critically ill: an overview of current cardiac output monitoring methods. F1000Res 2016. DOI: https://doi.org/ 10.12688/f1000research.8991.1.

3. Smallwood N, Dachsel M. Point-of-care ultrasound (POCUS): unnecessary gadgetry or evidence-based medicine? Clin Med (Lond) 2018; 18: 219-24. 
4. Abu-Zidan FM. Point-of-care ultrasound in critically ill patients: where do we stand? J Emerg Trauma Shock 2012; 5: 70-1.

5. Arntfield RT, Millington SJ. Point of care cardiac ultrasound applications in the emergency department and intensive care unit—a review. Curr Cardiol Rev 2012; 8: 98-108.

6. Naji A, Chappidi M, Ahmed A, Monga A, Sanders J. Perioperative point-of-care ultrasound use by anesthesiologists. Cureus 2021; DOI: https://doi.org/10.7759/cureus.15217.

7. Basmaji J, Ball I, Jones P, Rochwerg B, Arntfield R, CRIXUS Investigators (2020) Critical care ultrasonography in shock management: the elephant in Canadian intensive care units. Can J Anesth 67:1119-23.
8. Stickles SP, Carpenter CR, Gekle R, et al. The diagnostic accuracy of a point-of-care ultrasound protocol for shock etiology: a systematic review and meta-analysis. CJEM 2019; 21: 406-17.

9. Bughrara N, Renew JR, Alabre K et al (2022) Comparison of qualitative information obtained with the echocardiographic assessment using subcostal-only view and focused transthoracic echocardiography examinations. Can J Anesth. https://doi.org/10. 1007/s12630-021-02152-6

Publisher's Note Springer Nature remains neutral with regard to jurisdictional claims in published maps and institutional affiliations. 